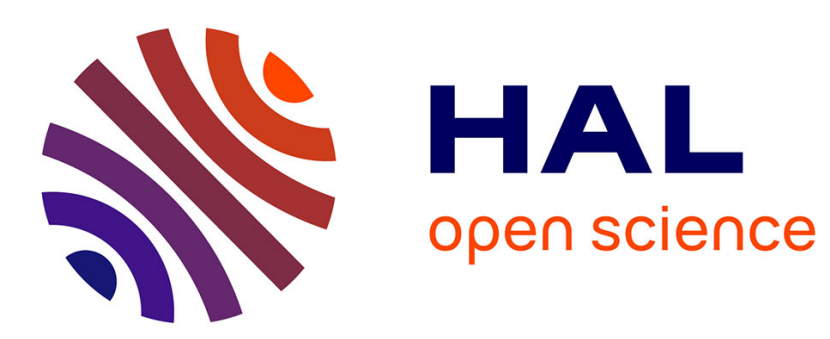

\title{
Periodic order induced by horizontal vibrations in a two-dimensional assembly of heavy beads in water
}

Régis Wunenburger, V. Carrier, Yves Garrabos

\section{To cite this version:}

Régis Wunenburger, V. Carrier, Yves Garrabos. Periodic order induced by horizontal vibrations in a two-dimensional assembly of heavy beads in water. Physics of Fluids, 2002, 14 (7), 2350 (10 p.). $10.1063 / 1.1483842$. hal-00719656

\section{HAL Id: hal-00719656 https://hal.science/hal-00719656}

Submitted on 1 Jul 2021

HAL is a multi-disciplinary open access archive for the deposit and dissemination of scientific research documents, whether they are published or not. The documents may come from teaching and research institutions in France or abroad, or from public or private research centers.
L'archive ouverte pluridisciplinaire HAL, est destinée au dépôt et à la diffusion de documents scientifiques de niveau recherche, publiés ou non, émanant des établissements d'enseignement et de recherche français ou étrangers, des laboratoires publics ou privés. 


\section{Periodic order induced by horizontal vibrations in a two-dimensional assembly of heavy beads in water}

Cite as: Physics of Fluids 14, 2350 (2002); https://doi.org/10.1063/1.1483842

Submitted: 27 December 2001 . Accepted: 17 April 2002 . Published Online: 03 June 2002

R. Wunenburger, V. Carrier, and Y. Garrabos

\section{ARTICLES YOU MAY BE INTERESTED IN}

The Dynamics of Spheres in Oscillatory Fluid Flows

AIP Conference Proceedings 1145, 1039 (2009); https://doi.org/10.1063/1.3179821

Measurements of the steady streaming flow around oscillating spheres using three dimensional particle tracking velocimetry

Physics of Fluids 20, 093304 (2008); https://doi.org/10.1063/1.2973204

Acoustic Streaming at Low Reynolds Numbers

The Journal of the Acoustical Society of America 25, 932 (1953); https://

doi.org/10.1121/1.1907221 


\title{
Periodic order induced by horizontal vibrations in a two-dimensional assembly of heavy beads in water
}

\author{
R. Wunenburger ${ }^{\text {a) }}$ \\ Laboratoire de Physique Statistique, Ecole Normale Supérieure, 24 rue Lhomond, \\ 75231 Paris Cedex 05, France \\ V. Carrier \\ Centre de Recherche Paul Pascal (UPR CNRS 8641), avenue Dr. A. Schweitzer, 33600 Pessac, France \\ Y. Garrabos \\ Institut de Chimie de la Matière Condensée de Bordeaux (UPR CNRS 9048), avenue Dr. A. Schweitzer, \\ 33608 PESSAC Cedex, France
}

(Received 27 December 2001; accepted 17 April 2002; published 3 June 2002)

\begin{abstract}
We report on the observation of the periodic pattern occuring in a two-dimensional (2D) assembly of heavy beads (of diameter $D=280-630 \mu \mathrm{m}$ ) immersed in water when the vessel containing the fluid and the beads is submitted to horizontal vibrations (of frequency $f$ and oscillation amplitude $A$ ). Under vibrations, regularly spaced, one bead thick lines perpendicular to the direction of vibrations are formed at small surface fraction. In the ranges $f=10-20 \mathrm{~Hz}$ and $A=0.7-3.5 \mathrm{~mm}$, the amplitude of the oscillatory motion of the beads relative to the fluid $B$ is found to vary linearly with $A$. A simple phenomenological model based on dynamic solid friction and viscous drag gives qualitatively account for this linear dependence. The period of the pattern $\lambda$ is found to vary with viscosity $v$, $\omega=2 \pi f, D$, and $B$ according to the law $\lambda / D \sim(B / D)^{0.5}(B \omega D / v)^{-0.21}$. The dynamics of pattern formation exhibits several scenarii, depending on the initial bead distribution, as well as several characteristic time scales. We review and discuss possible mechanisms of pattern formation reported in literature and proposed for similar phenomena observed in the frame of rheology of suspensions and acoustic streaming flows. (C) 2002 American Institute of Physics. [DOI: 10.1063/1.1483842]
\end{abstract}

\section{INTRODUCTION}

The behavior of macroscopic solid particles immersed in a fluid is determined by collisions, solid friction, and hydrodynamic interactions, i.e., reciprocal effects between particle motion and induced fluid flow. Hydrodynamic interactions are involved in many physical phenomena, e.g., stability and sedimentation of colloidal or macroscopic suspensions, multiphase transport, mixing and segregation of particles in flows. Applying external force fields to suspensions was found to induce hydrodynamic interactions that are helpful to control the distribution, the orientation or the size of particles in the fluid. The effects of externally imposed flows on suspensions are reviewed by Petit. ${ }^{1}$ The effect of shear on the structure of colloidal suspensions ${ }^{2,3}$ has been evidenced long ago. In macroscopic suspensions, a shear induced crystallization phenomenon was evidenced when an alternating shear stress was applied (Ref. 4 and references therein). In more complex flows involving inhomogeneous shear stress distribution, like flows in tubes ${ }^{5}$ or in planar shear cells, ${ }^{6}$ the phenomenon of migration of neutrally buoyant dense suspensions from regions of high shear rate to regions of low shear rate was identified. More recently, particle segregation in neutrally buoyant macroscopic suspensions partially filling a horizontal Couette shear cell and a horizontal cylinder was

\footnotetext{
a) Author to whom correspondence should be addressed. Phone: (33) 14432
} 35 01; fax (33) 1443234 33; electronic mail: wunenbur@1ps.ens.fr reported. $^{7,8} \mathrm{We}$ are interested in the action of alternating flows on suspensions through nonlinear effects (streaming flows). The specificity of alternating flows is that their linear effects have zero time-averaged value. Thus, only nonlinear effects of these flows are expected to be visible, even if they are small compared to the instantaneous linear effects. A striking effect of streaming flows was early reported by Andrade and Lewer ${ }^{9}$ and by Carrière: ${ }^{10}$ When powder is laid into an air tube in which a standing acoustic wave is established (Kundt's tube experiment), powder heaps form at the nodes whereas periodic distributions of one particle thick lines perpendicular to the tube form at the antinodes. ${ }^{9}$ In this article we report on the occurrence of a periodic distribution of regularly spaced one bead thick lines perpendicular to the direction of vibrations when a monolayer of heavy beads immersed in water are set mechanically into vibration. To our knowledge, this phenomenon has not been previously reported. Moreover, since to our knowledge no theoretical prediction has been proposed concerning this ordering phenomenon, we give numerous quantitative as well as qualitative details on the observed phenomenon as well as numerous hypotheses relative to the possible mechanisms. In particular, we compare our observations to the phenomenon observed in a Kundt's tube.

In Sec. II, we present the experimental setup. In Sec. III we present the main features of the phenomenon of periodic ordering presented above as well as our measurements of the periodicity of the periodic pattern. Since this periodic order 


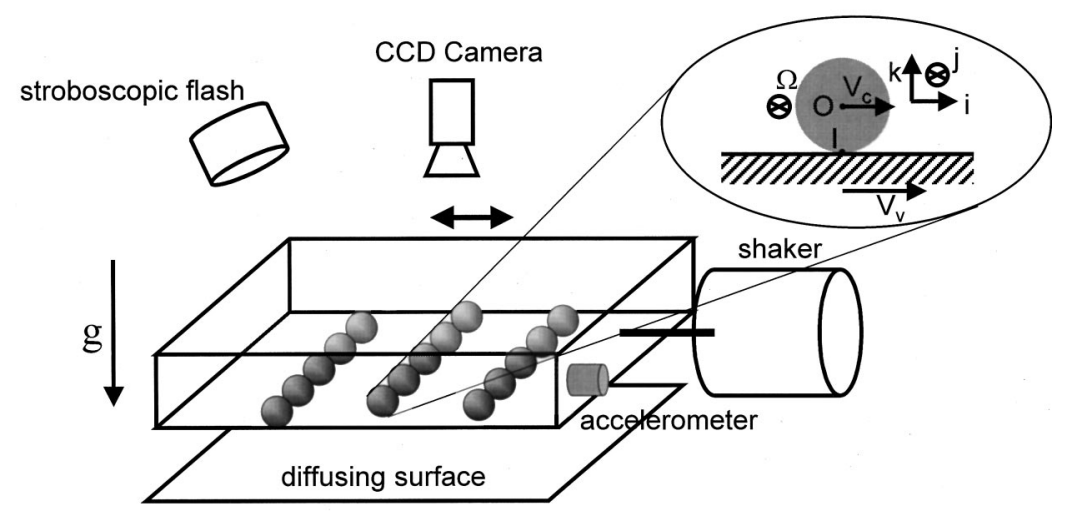

FIG. 1. Experimental setup. Inset: Conventions for the dynamics of a sphere rolling with solid friction on an oscillating wall.

is determined by the oscillatory motion of the beads, we present in Sec. IV our measurements of the amplitude of the oscillatory motion of the bead lines, and discuss their dynamics. Using the experimental results presented in Sec. IV, we determine in Sec. V an experimental law of variation for the periodicity of the pattern. In Sec. VI we discuss various mechanisms of hydrodynamic interactions found in the litterature which could explain the observed pattern. In Sec. VII we finally give some qualitative information on the pattern formation dynamics and defects.

\section{EXPERIMENTAL SETUP}

The experimental setup is schematized in Fig. 1. It is composed of spherical bronze beads (of typical diameter $D$ $=0.3 \mathrm{~mm}$ ) lying on the bottom of a $100 \mathrm{~mm} \times 100 \mathrm{~mm}$ $\times 10 \mathrm{~mm}$ vessel. The top and bottom walls of the vessel, of transparent altuglas, allow for the observation of the bead distribution by light transmission. Special care is taken in order to insure horizontality of the bottom wall of the cell. The cell is then completely filled with gas-free water, so that there is no free water-air interface (special care is taken in order to avoid the presence of air bubbles). The vessel is vibrated horizontally with a Bruel and Kjaer (BK) 4809 shaker powered by a BK 2706 Power Amplifier driven by a sinusoidal signal produced by a Wavetek 81 Function Generator. Noting $x$ the position of any point of the vessel along an horizontal axis and $t$ the time, the horizontal sinusoidal acceleration applied to the vessel reads $\ddot{x}(t)=-\Gamma \sin (\omega t)$, where $\omega=2 \pi f$ is the pulsation and $f$ the frequency. This acceleration is measured by a BK 4393 accelerometer powered by a BK Nexus Charge Amplifier and visualized on an Tektronix TDS 220 Digital Oscilloscope. The position of the vessel $x$ reads $x(t)=A \sin (\omega t)+x_{0}$, where $A=\Gamma / \omega^{2}$. The frequencies and amplitudes investigated are $f=10,15$, and 20 $\mathrm{Hz}$ and $A=0.5-3.7 \mathrm{~mm}$. The bead distribution is observed using a Lutron DT2239-II stroboscopic flash light of frequency slightly different from the vibration frequency in order to observe the whole phase of the motion. The pictures are recorded at the VHS format at a frequency of $25 \mathrm{~Hz}$ using a Pulnix PE 2015 CCD camera, then digitized. A $5 \mathrm{~mm} \times 5 \mathrm{~mm}$ grid printed on the bottom of the vessel gives a scaling of the picture to be determined. The resolution of the pictures is typically 12 pixels per millimeter.

\section{STEADY PERIODIC PATTERN}

Three different sieved bead samples were used, characterized by bead density $d=7.8$ and diameter range $D$ $=280-315 \mu \mathrm{m}, D=355-400 \mu \mathrm{m}$, and $D=500-630 \mu \mathrm{m}$ (in the following analysis of the experimental data the average values of the bead diameters are considered, $D=297$, 377 , and $565 \mu \mathrm{m})$. The number of beads was chosen so that the fraction of the surface covered by the beads to the surface of the vessel bottom $\phi_{S}$ be small and constant (7\%). In order to favor initially strong repulsive interactions between beads, their initial distribution is always chosen to be a compact monolayer of beads in contact, located in the middle of the vessel. Two types of initial distributions were tested, a square of side $\simeq 30 \mathrm{~mm}$, and a stripe perpendicular to the direction of the acceleration. When vibrations are applied, after a transient lasting for a few tens of seconds, regardless of their initial compact distribution, the beads form regularly spaced lines (distant of the period $\lambda$ ), as shown in Fig. 2. The lines are perpendicular to the direction of vibrations. The characteristic features of the steady state of the periodic pattern under vibrations are the following. A line of beads is only

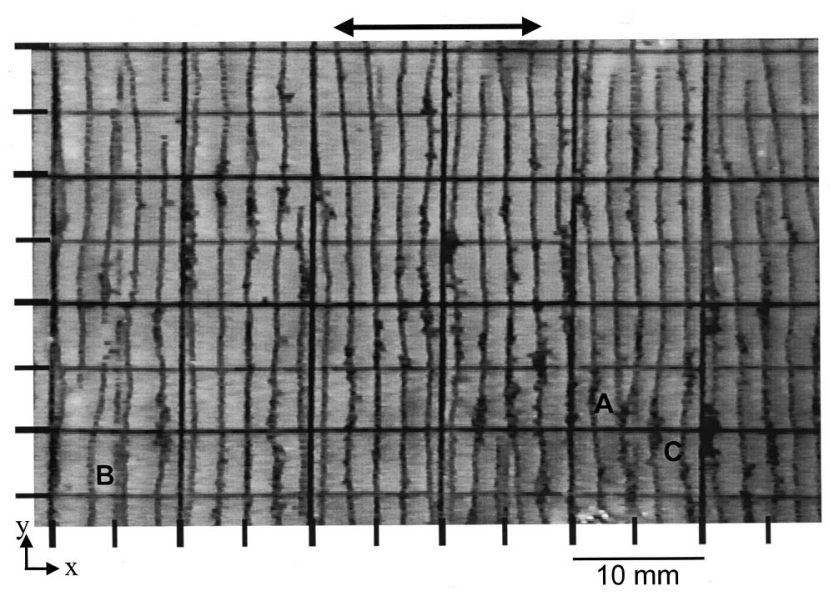

FIG. 2. Picture of steady periodic pattern formed by a 2D assembly of bronze beads of diameter $D=355-400 \mu \mathrm{m}$ immersed in water when submitted to horizontal sinusoidal vibrations at frequency $f=20 \mathrm{~Hz}$ and acceleration amplitude $\Gamma=18.7 \mathrm{~m} \cdot \mathrm{s}^{-2}$. The black ticks locate the grid lines printed on the bottom of the vessel, $5 \mathrm{~mm}$ spaced. Double arrow indicates the direction of oscillations. A: fork defect. B: local pattern dilation. C: line thickening. 


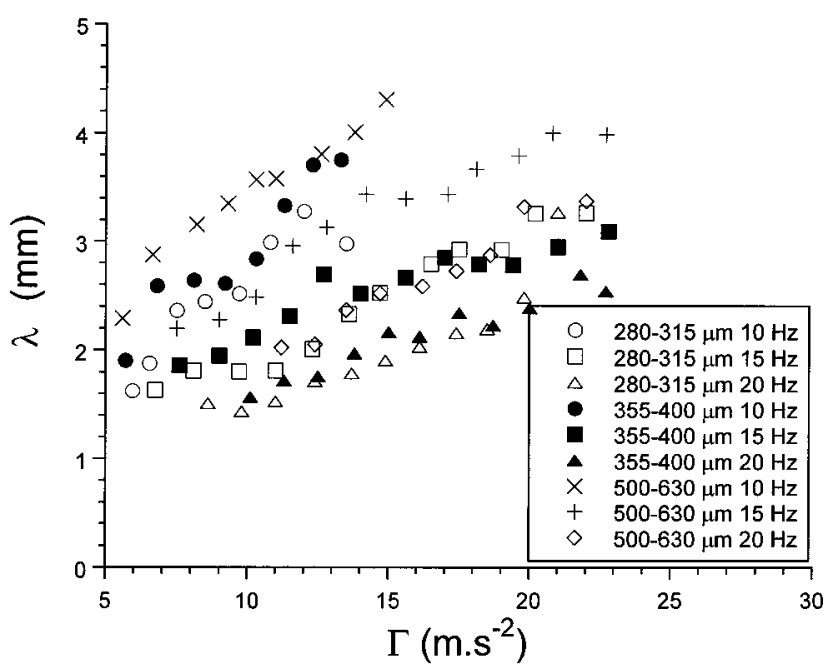

FIG. 3. Variations of periodicity $\lambda$ of the pattern as a function of the acceleration $\Gamma$ for various bead diameters and frequencies.

one bead thick. Along a line, the beads are either in contact or very close to each other (distance much smaller than $D$ ). The periodic pattern oscillates as a whole ( $\lambda$ remains constant) at the vibration frequency $f$ in the reference frame of the vessel. Since all the beads oscillate in phase with each other and with the same amplitude, two neighboring lines never collide. $\lambda$ depends on both $D, A$, and $f$, but is independent of $\phi_{S}$ for small values of $\phi_{S}$. The steady pattern does not depend on the initial distribution of the beads, only the transients and their duration do: The same steady pattern of period $\lambda_{1}$ can be obtained for the vibrational parameters $\left(A_{1}, f\right)$ either from an initial dense distribution as described above, or from another steady pattern of period $\lambda_{2}>\lambda_{1}$ obtained for $\left(A_{2}, f\right)$ with $A_{2}>A_{1}$ ( $\lambda$ increases with increasing $A)$. The fact that $\lambda$ be independent of $\phi_{S}$ means that (i) the periodic pattern does not cover the whole surface of the vessel at small enough $\lambda$, and (ii) when the periodic pattern covers the whole surface, some of the beads stick to the sides of the vessel so that the number of lines be reduced. The period of the pattern $\lambda$ was measured for various values of parameters $D, A, f . \lambda$ is determined by averaging the distance between lines on the whole pattern. The variations of $\lambda$ as a function of the acceleration $\Gamma$ are plotted in Fig. 3 for various values of $D$ and $f$. $\lambda$ increases with increasing $\Gamma$ at fixed $D$ and $f$ and decreases with increased $D$ and $f$ at fixed $\Gamma$. In order to clarify such behaviors, we now study the oscillatory motion of the bead lines.

\section{MOTION OF THE BEADS RELATIVE TO THE FLUID}

Since the acoustic wavelength in water corresponding to $f=10-20 \mathrm{~Hz}$ is much larger than the vessel size, the periodic pattern can obviously not be due to compressible flows. When the two-phase system is submitted to an horizontal acceleration, because of their density larger than water, beads move with respect to the fluid as in the case of sedimentation. Under the effect of an oscillatory acceleration, beads oscillate in the frame of reference of the fluid. The flow induced by these oscillations induces in turn hydrodynamic interactions between the beads. It is noteworthy that in absence of beads no fluid motion occurs in the vessel frame of reference. As a consequence the hydrodynamic interactions between beads are only due to the motion of the beads with respect to the vessel and not to the influence of any externally imposed flow. Since $\lambda$ does not depend on $\phi_{S}$, these induced hydrodynamic interactions consist in (i) an attractive force between the beads along a line, and both (ii) a repulsive force between the lines at short distance and (iii) an attractive force between the lines at long distance. Thus, the key parameter of the formation of the periodic pattern is not the motion of the fluid relative to the laboratory, but rather the motion of the beads relative to the medium which causes their interactions, i.e., to the fluid. This part is dedicated to the study of the motion of the beads relative to the fluid. We first present our measurements of the oscillatory motion of the bead lines.

\section{A. Measurement of the motion}

An easily measurable quantity is the amplitude $B$ of the oscillatory motion of the beads relative to the fluid noted $y(t)=B S(t)+y_{0}$, where $S$ is an unknown periodic function of time of extreme values 1 and -1 , and $y_{0}$ is a constant depending on the line considered. It is possible to detect with the naked eye on the video pictures the two extreme positions of the bead lines relative to the grid, i.e., two instants $t_{1}$ and $t_{2}$ at which $S\left(t_{1}\right)=1, S^{\prime}\left(t_{1}\right)=0$ and $S\left(t_{2}\right)=-1$, $S^{\prime}\left(t_{2}\right)=0$. For this we establish a slight frequency shift between the stroboscopic flash and the shaker that slowly changes the phase shift between the alternating motion and the flash. This simple detection is facilited by the fact that the beads spend a lot of time per cycle at the extreme positions of their oscillating trajectory, where their velocity cancels [the histogram of $x(t)=B \sin (\omega t)$ diverges for $x= \pm B$ ]. The quantity $2 B$ is measured by intercorrelating the pictures taken at $t_{1}$ and $t_{2}$. Noting $I(x, y, t)$ the value of the gray level of pixel of coordinates $(x, y)$ on picture taken at time $t$, (coordinates are defined in Fig. 2), the intercorrelation function of pictures taken at $t_{1}$ and $t_{2}$ is defined as $\left\langle\int I\left(x, y, t_{1}\right) I(x\right.$ $\left.\left.+\Delta x, y, t_{2}\right) d x\right\rangle_{y}$, where $\Delta x$ is the shift between the two pictures in the vibration direction, and \langle\rangle$_{y}$ is the average on all $y$ values. In Fig. 4 a series of intercorrelation functions of pairs of pictures taken during experiments performed at $f$ $=10 \mathrm{~Hz}$ and at various values of $A$ is plotted. The distance between the correlation maxima due to the grid lines (dashed curve on the left) and the correlation maxima due to the bead lines (dashed curve on the right) is equal to $2 B$. The variations of $B$ as a function of $A$ for various values of $f$ and $D$ are plotted in Fig. 5. $B$ is found to vary roughly linearly with $A$ ( $B \simeq \alpha\left(A-A_{0}\right)$ ) with $\alpha$ and $A_{0}$ depending on both $f$ and $D$. The high-frequency limit of our experimental investigation was fixed equal to $20 \mathrm{~Hz}$ mainly because our shaker did not allow us to realize values of $B$ sufficiently large to be detectable with our detection method at larger frequencies. Nevertheless, the periodic pattern could be observed at frequencies up to $120 \mathrm{~Hz}$.

It is noteworthy that isolated beads were found to oscillate with the same phase and amplitude than the bead lines. 


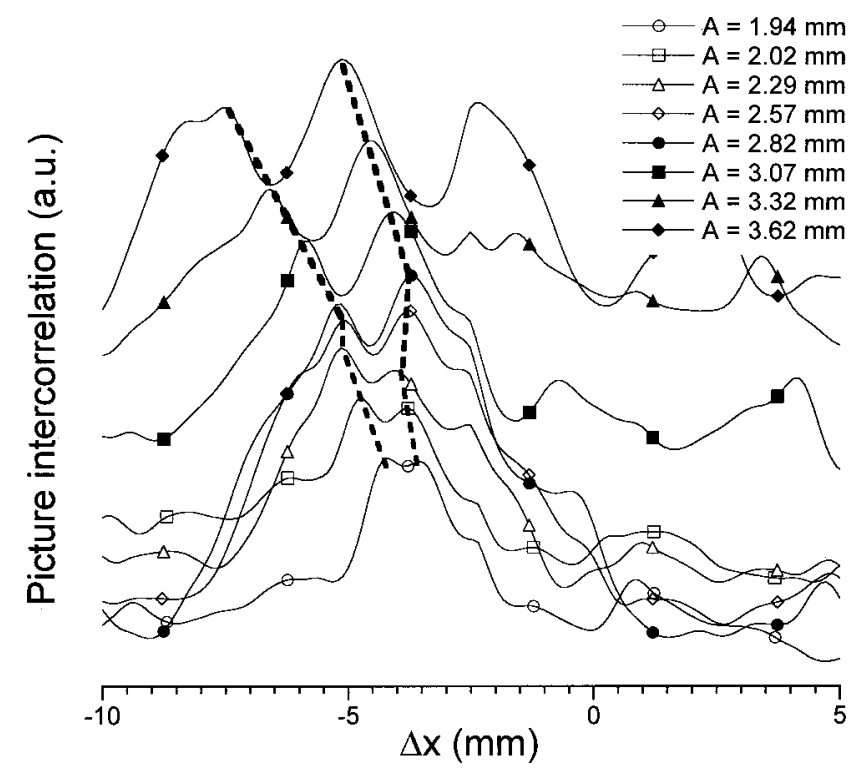

FIG. 4. Series of intercorrelation functions of pairs of pictures taken during experiments performed at $f=10 \mathrm{~Hz}$ and at various values of $A$. The curves were shifted vertically for clarity (vertical scale is arbitrary). $\Delta x$ is the shift between the intercorrelated pictures along the direction of vibrations. The distance between the correlation maxima due to the grid (dashed curve on the left) and the correlation maxima due to the bead lines (dashed curve on the right) is equal to $2 B$, i.e., twice the amplitude of oscillation of the beads with respect to the fluid.

This observation justifies that the dynamics of the bead lines can be analyzed in terms of the dynamics of a single bead. Moreover it shows that only the dimension of the oscillating object in the direction of vibrations matters in this ordering phenomenon. We now discuss the forces that apply on a single rolling bead and model the oscillatory motion of the bead lines described above.

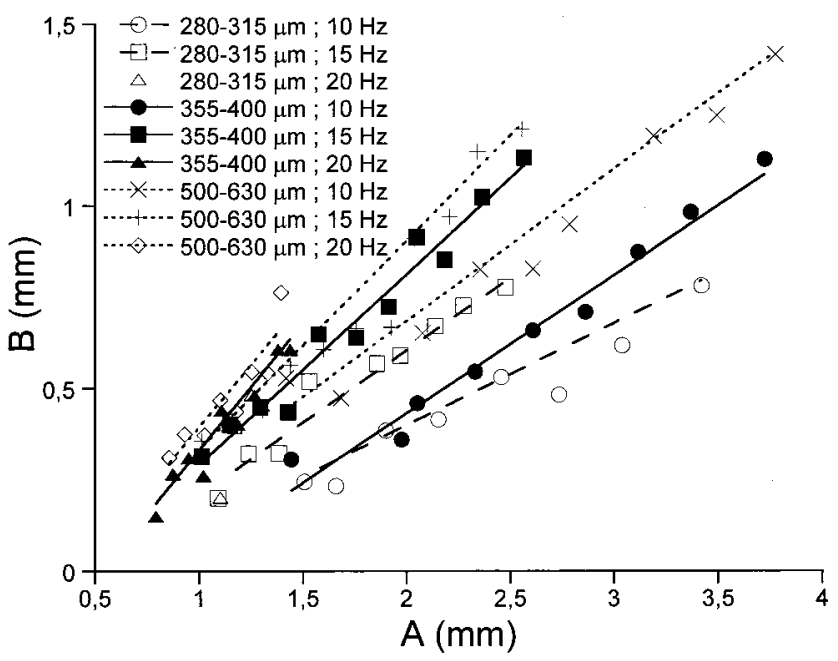

FIG. 5. Variations of amplitude of oscillation of bead lines relative to the fluid $B$ as a function of fluid oscillation amplitude $A$, for various values of bead diameter range $D$ and of oscillation frequency $f$. Symbols: Experimental data. Lines: Best fits obtained using Eq. (11) with $k$ and $\mu_{D}$ as free parameters (see Table I for the best fit values).

\section{B. Model of the motion}

Since in the frequency range $f=10-20 \mathrm{~Hz}$ no compressible flow occurs in the fluid, in absence of beads the pressure field $p$ in the fluid is at any time hydrostatic, i.e., $\nabla p(t)$ $=\rho_{f}\left(-d \mathbf{V}_{v} / d t+\mathbf{g}\right)$, where $\rho_{f}$ is the fluid density, $\mathbf{V}_{v}$ is the vessel velocity, $\mathbf{g}$ is Earth gravity, and the fluid is at rest in the frame of reference of the vessel. At low Reynolds number, the resulting effect of pressure and viscous forces on a single bead can be modeled as (i) the acceleration that would apply to a fluid particle replacing the bead, ${ }^{11}$ (ii) the drag force and torque induced by its motion relative to the fluid as considered as stationary, ${ }_{12}^{12}$ (iii) an added mass, ${ }^{13}$ (iv) a socalled "historic" force, ${ }^{13}$ and torque. ${ }^{14}$ An additional force applying on the bead is the solid friction with the wall.

The first question to be addressed is whether the bead does roll without slipping on the bottom or not. Unfortunately, the added mass as well as the historic force and torque were not calculated for the present case of a bead touching a wall, but for the case of a bead in an unbounded fluid. Consequently, no exact prediction can be established for our case. Moreover, the Reynolds number associated to the flow around the bead $\operatorname{Re}=\omega B D / v$ (where $\omega B$ is the typical velocity of the bead relative to the fluid, and $v$ is the fluid kinematic viscosity) is not small compared to unity ( $\operatorname{Re}$ ranges from 2 to 50 in our experiment), although not large. Nevertheless, retaining only the drag force and torque applying at low Re on the bead near the wall as established by Goldman et al. using lubrication theory ${ }^{12}$ should provide a pertinent order of magnitude for the slipping threshold.

The main feature of the lubrication theory is that the force and torque diverge slowly when the distance $e$ between the bead and the wall vanish (as the logarithm of $e / D$ ). In order to account for both solid friction (implying contact between the bead and the wall) and finite drag force and torque (implying a finite distance between the bead and the wall), the roughness of the surfaces in the contact area is usually considered as permitting both the fluid flow around its roughness elements and solid contact between the surface and the roughness elements. Thus, the pertinent distance between the bead and the wall to be considered is of the order of the roughness of the surfaces, typically some microns (or tens of microns). The validity of these assumptions and of the prediction of Goldman et al. was recently quantitatively demonstrated by Smart et al. in the particular case of a sphere rolling down steadily on an inclined wall in a viscous fluid. ${ }^{15}$ For the case of our experiment, assuming a quasisteady creeping flow around the oscillating bead, solid friction without slip, and using the geometrical conventions given in the insert of Fig. 1, in the frame of reference of the laboratory the bead motion is governed by the following set of equations:

$$
\begin{aligned}
& m_{b} \frac{d \mathbf{V}_{c}}{d t}=m_{f} \frac{d \mathbf{V}_{v}}{d t}+\mathbf{F}_{S}+\mathbf{F}_{T}+\mathbf{F}_{R}, \\
& \frac{1}{10} m_{b} D^{2} \frac{d \boldsymbol{\Omega}}{d t}=\mathbf{C I} \wedge \mathbf{F}_{S}+\mathbf{T}_{T}+\mathbf{T}_{R}, \\
& \mathbf{V}_{c}+\mathbf{\Omega} \wedge \mathbf{C I}=\mathbf{V}_{v} .
\end{aligned}
$$


$m_{b}$ is the bead mass, and $m_{f}$ the mass of a fluid particle replacing the bead. $\mathbf{V}_{c}=V_{c} \hat{\imath}$ is the velocity of the bead gravity center $C$, and $\boldsymbol{\Omega}=\boldsymbol{\Omega} \hat{\jmath}$ is the angular velocity of the bead around its horizontal symmetry axis normal to the direction of vibrations. $I$ is the bead-wall contact point. $\mathbf{F}_{S}$ is the static friction force which is opposite to $\mathbf{V}_{c}-\mathbf{V}_{v}$ and verifies

$$
\left\|\mathbf{F}_{S}\right\| \leqslant \mu_{S}\left(m_{b}-m_{f}\right)\|\mathbf{g}\|,
$$

with $\mu_{S}$ the static friction coefficient. $\mathbf{F}_{T}, \mathbf{F}_{R}$, (resp. $\mathbf{T}_{T}$ and $\mathbf{T}_{R}$ ) are the viscous forces (resp. torques) due to the translational $(T)$ and rotational $(R)$ velocities of the bead relative to the fluid $^{12}$

$$
\begin{aligned}
\mathbf{F}_{T} & =F_{T}^{*} 3 \pi D \rho_{f} \nu\left(\mathbf{V}_{c}-\mathbf{V}_{v}\right), \\
F_{T}^{*} & =\frac{8}{15} \ln \left(\frac{2 e}{D}\right)-0.9588<0, \\
\mathbf{T}_{T} & =T_{T}^{*} 4 \pi D^{2} \rho_{f} \nu \hat{\mathbf{k}} \wedge\left(\mathbf{V}_{c}-\mathbf{V}_{v}\right), \\
T_{T}^{*} & =-\frac{1}{10} \ln \left(\frac{2 e}{D}\right)-0.1895>0, \\
\mathbf{F}_{R} & =F_{R}^{*} 3 \pi D \rho_{f} \nu \mathbf{\Omega} \wedge \hat{\mathbf{k}}, F_{R}^{*} \\
& =-\frac{2}{15} \ln \left(\frac{2 e}{D}\right)-0.2526>0, \\
\mathbf{T}_{R} & =T_{R}^{*} D^{3} \rho_{f} \nu \boldsymbol{\Omega}, F_{T}^{*}=\frac{2}{5} \ln \left(\frac{2 e}{D}\right)-0.3817<0 .
\end{aligned}
$$

Under the hypothesis of creeping flow, the bead oscillation is sinusoidal. In complex notation, the general solution of the linear system (1)-(3) expressed under the form of the bead velocity relative to the fluid $V=\left(\mathbf{V}_{c}-\mathbf{V}_{v}\right) \cdot \hat{\imath}$ is

$$
V=-\frac{\rho_{b}-\rho_{f}}{\rho_{b}} \frac{1}{\frac{7}{5}+i S t^{-1}\left(F_{T}^{*}+\frac{4}{3} T_{T}^{*}+F_{R}^{*}+\frac{4}{3} T_{R}^{*}\right)} A \omega,
$$

where $S t$ is the Stokes number associated to the bead motion $S t=(1 / 18)\left(\rho_{b} / \rho_{f}\right)\left(D^{2} \omega / v\right)$. The no-slip condition (4) can be expressed as

$$
\Gamma \leqslant\left|\frac{\frac{7}{5}+i S t^{-1}\left(F_{T}^{*}+\frac{4}{3} T_{T}^{*}+F_{R}^{*}+\frac{4}{3} T_{R}^{*}\right)}{\frac{2}{5} i-\frac{4}{3} S t^{-1}\left(T_{R}^{*}+T_{T}^{*}\right)}\right| \mu_{S} g .
$$

The upper value of the right-hand term of Eq. (10), as obtained asympotically for $S t \gg 1$, is $3.5 \mu_{S} g$. In absence of any measurement of $\mu_{S}$ for bronze-altuglass in water, we use the value $\mu_{S}=0.1$, which is typical of well-lubricated metalnonmetal contacts, i.e., $0.05-0.12 .{ }^{16}$ In Fig. 6 the variations of the slipping acceleration threshold [as defined as the equality between both sides of Eq. (10)] as a function of $D$ are plotted for various values of $f$ and two typical values of the roughness $e=10 \mu \mathrm{m}$, and $e=1 \mu \mathrm{m}$. In the range of moderate values of $S t$ (2.5-17.5) explored in our experiment, the value of the slipping acceleration threshold varies between $2.4 \mathrm{~m} \cdot \mathrm{s}^{-2}$ and $3.4 \mathrm{~m} \cdot \mathrm{s}^{-2}$. These values are below the smallest value $5.6 \mathrm{~m} \cdot \mathrm{s}^{-2}$ of $\Gamma$ at which the periodic pattern is observed (see Fig. 1).

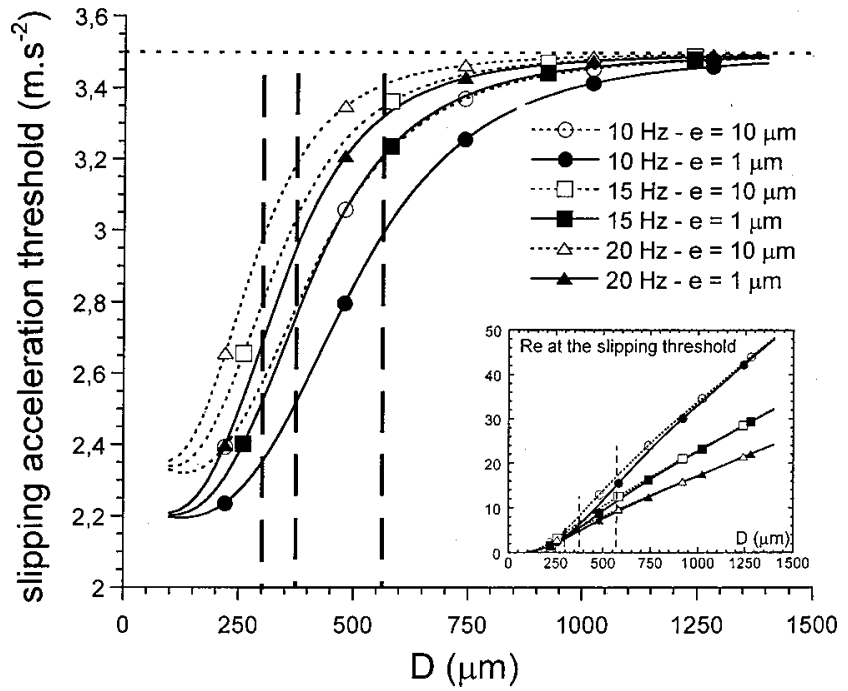

FIG. 6. Acceleration threshold of the wall beyond which slipping occurs (under assumption of quasi-steady creeping flow) as a function of sphere diameter $D$ for various oscillation frequencies $f$ and of sphere roughness characteristic length scale $e$.

We now estimate the magnitude of the corrections to Eqs. (1) and (2). For the case of a sphere oscillating in an unbounded fluid (i) the added mass, $\frac{1}{2} m_{f}$, is small compared to $m_{b}$, and (ii) the ratio of the historic force $\frac{3}{2} \pi \rho_{f} v D^{2}$ $\int_{0}^{t}\left(d \mathbf{V}_{c} / d \tau\right)(t-\tau)^{-1 / 2} d \tau$ to the steady drag force $-3 \pi D \rho_{f} v \mathbf{V}_{c}$ is ${\sqrt{D^{2} \omega / 8 v}}^{13}$ and ranges from 0.8 to 2.2 in the frequency and diameter ranges corresponding to our experiment. One can infer that the corresponding historic forces and torques for the case of a sphere oscillating near a wall may also be of the same order of magnitude as the lubrication forces and torques as defined in Eqs. (5)-(8). As a consequence, the prediction 10 is not quantitatively accurate, but should provide a good order of magnitude for the actual slipping threshold. We thus retain from this analysis that in our experiment the beads should slip on the bottom wall during a large part of the oscillation period.

The second problem to be addressed is the reason for the observed variations of $B$ with $A, f$ and $D$. We propose a simple, phenomenological model in which only the translational degree of freedom of the bead is retained and permanent slip of the bead on the wall is assumed. The retained forces are the viscous drag force $\mathbf{F}_{T}=-k 3 \pi D \rho_{f} \nu\left(\mathbf{V}_{c}\right.$ $-\mathbf{V}_{v}$ ) ( $k$ accounts for an increase of the drag force due to lubrication and inertia effects, is of the same order of magnitude as $F_{T}^{*}$, and is a free parameter of the model) and a dynamic solid friction force $\mathbf{F}_{D}=-\mu_{D}\left(m_{b}-m_{f}\right)\|\mathbf{g}\|\left(\mathbf{V}_{c}\right.$ $\left.-\mathbf{V}_{v}\right) /\left(\left\|\mathbf{V}_{c}-\mathbf{V}_{v}\right\|\right)$ that applies at any time $\left(\mu_{D}\right.$ is the dynamic friction coefficient and is also a free parameter of the model, considering the simplicity of the friction model). The corresponding dynamic equation for the velocity of the bead relative to the fluid is

$$
\dot{V}+k \omega S t^{-1} V+\mu_{D} g \frac{\rho_{b}-\rho_{f}}{\rho_{b}} \operatorname{Sign}(V)=-\frac{\rho_{b}-\rho_{f}}{\rho_{b}} \dot{V}_{v} .
$$

Since the slipping threshold is neglected, this model is obvi- 
TABLE I. Values of the free parameters $k$ and $\mu_{D}$ corresponding to the best fit of the model Eq. (11) to the experimental data. The corresponding best fits are plotted together with the experimental data in Fig. 5.

\begin{tabular}{|c|c|c|c|c|c|c|}
\hline \multirow{2}{*}{$\begin{array}{c}f(\mathrm{~Hz}) \\
D(\mu \mathrm{m})\end{array}$} & \multicolumn{2}{|c|}{10} & \multicolumn{2}{|c|}{15} & \multicolumn{2}{|c|}{20} \\
\hline & $k$ & $\mu_{D}$ & $k$ & $\mu_{D}$ & $k$ & $\mu_{D}$ \\
\hline $280-315$ & 7 & 0.17 & 7 & 0.33 & $\cdots$ & $\ldots$ \\
\hline $355-400$ & 8 & 0.26 & 7.75 & 0.33 & 6 & 0.8 \\
\hline $500-630$ & 16 & 0.11 & 14.3 & 0.33 & 14 & 0.65 \\
\hline
\end{tabular}

ously pertinent for $\mu_{D} g \ll \Gamma$ and fails for $\mu_{D} g>\Gamma$. When existing, the numerically computed solution of Eq. (11) exhibits a periodic sine-like solution after a short transient. The resulting relation between $B$ and $A$ is found to be linear $[B$ $\left.=\alpha\left(A-A_{0}\right)\right]$ with $A_{0} \neq 0$. The values of $k$ and $\mu_{D}$ corresponding to the best fit of the model to the experimental data are given in Table I, and the corresponding best fits are plotted together with the experimental data in Fig. 5 (lines). The limits of accuracy of this phenomenological model are obvious when considering the variations of $\mu_{D}$ with $D$ and in particular with $f$, which have no physical grounds. Moreover, the mean value of $\mu_{D},\left\langle\mu_{D}\right\rangle=0.37$, although physically acceptable, is much larger than the values reported in Ref. 16.

As a conclusion of this part, slip is likely to occur during a large part of an oscillation cycle. In other words, rotation seems to matter less than translation in this ordering phenomenon. The qualitative model presented above shows that solid dynamic friction together with fluid drag force can explain the dependence of $B$ with respect to $A$ and $f$. Since the theoretical determination of actual historic forces and torques applying on a translating and rotating sphere near a wall are extremely difficult, even when $\operatorname{Re} \ll 1$, the improvement of such an analysis is unlikely. A further step would be to increase the precision of the measurement of the bead motion relative to the fluid and to meadure the bead rotation, e.g., using a high-speed camera. The measurement of the phase shift between the oscillation of the bead and the oscillation of the fluid, which is visible with the naked eye, could also be exploited.

Once the amplitude of the oscillatory motion $B$ being measured, we can now seek an experimental law of variation for the periodicity of the pattern $\lambda$ using $B$ instead of $A$ as a pertinent length scale.

\section{AN EXPERIMENTAL LAW OF VARIATION FOR $\lambda$}

We first have to define the pertinent dimensionless quantities that determine $\lambda$. Since $\lambda$ does not depend on $\phi_{S}$ at small $\phi_{S}$, we assume that the horizontal (longitudinal and transverse) dimensions of the vessel are not pertinent length scales. Because of the small value of the ratio of the bead diameter $D$ to the vessel height $H(0.03-0.06)$ we also assume that $D / H$ is neither a pertinent quantity. As a consequence, $\lambda$ depends only on $D, B, \omega$, and $v$. We choose to determine the relation between the dimensionless quantities $\lambda / D, B / D$ and $\operatorname{Re}=B \omega D / v$. For this purpose, we assume a scaling law of the form

$$
\frac{\lambda}{D} \sim\left(\frac{B}{D}\right)^{x} \operatorname{Re}^{y} .
$$

Instead of using the experimental values of $B$, which present a large dispersion, we rather fit linear laws $B=\alpha\left(A-A_{0}\right)$ to the experimentally measured $B$ values and then use these laws to calculate $B$ for each value of $A$. We then determine the values of $x$ and $y$ giving the best fit to the data $\{B(A), \lambda(A)\}: x=0.50$ and $y=-0.21$. To check the validity of this fit, the variation of $\lambda / D(B / D)^{-1 / 2}$ as a function of Re is plotted in $\log -\log$ scales in Fig. 7, whereas the variation of $(\lambda / D) \mathrm{Re}^{0.21}$ as a function of $B / D$ is plotted in $\log -\log$ scales in Fig. 8. Although no theoretical argument supports the hypothesis of scaling law, and given the precision of the determination of $B$, we judge the agreement between the experimental data and the scaling law fit as satisfactory, at least in validating the dimensional analysis proposed above. Its is noteworthy that the density contrast $\left(\rho_{b}-\rho_{f}\right) / \rho_{b}$, which is presumably also a pertinent quantity, was not varied in these experiments. Finally, we mention that such a simple scaling law evidently fails if the formation of the pattern is found to occur above a given threshold of any flow control parameter. This eventuality as well as the various mechanisms of hydrodynamic interactions which could explain the observed pattern are discussed in the next part.

\section{DISCUSSION ABOUT A POSSIBLE ORDERING MECHANISM}

In this part, we review some mechanisms that could explain the periodic order observed in our experiment. The fact that $\lambda$ does not depend on $\phi_{S}$, as well the law of variation of $\lambda$ with respect to $A / D$ and $\mathrm{Re}$, indicate that the periodic order does not result only from a repulsion between lines but

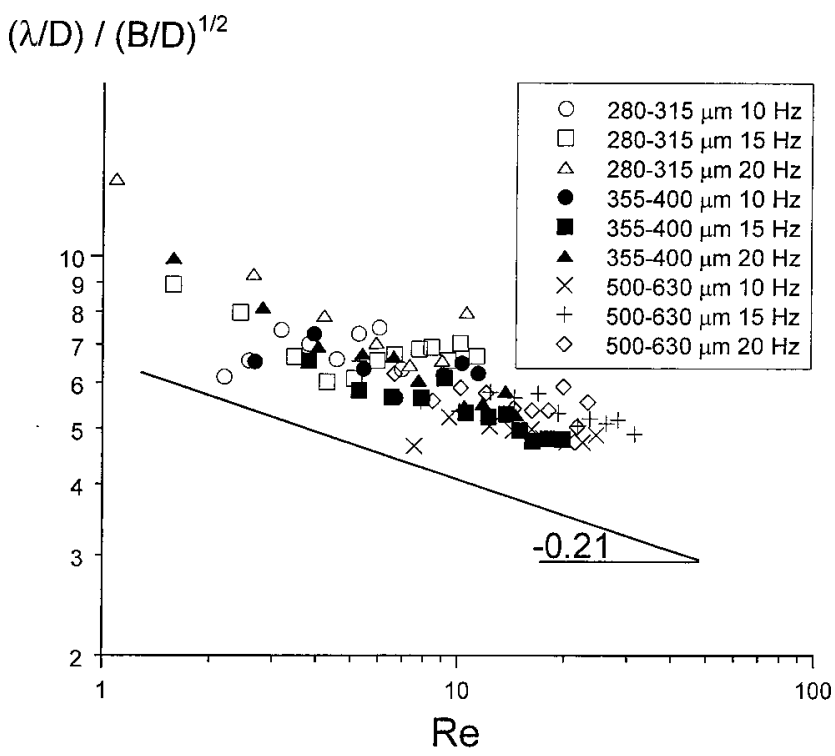

FIG. 7. Variation (in $\log -\log$ scales) of dimensionless pattern period $\lambda / D$ reduced with square root of dimensionless amplitude of oscillation of the bead line relative to the fluid $B / D$ as a function of Reynolds number Re for various values of bead diameter range $D$ and of frequency $f$. 


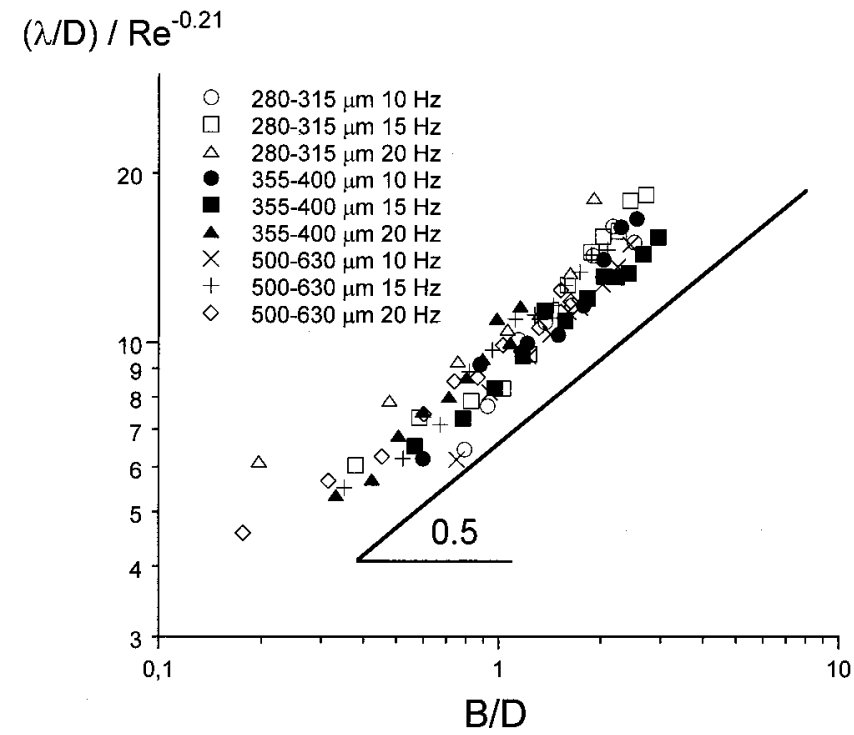

FIG. 8. Variation (in $\log -\log$ scales) of dimensionless pattern period $\lambda / D$ reduced with $\mathrm{Re}^{-0.21}$ as a function of dimensionless oscillation amplitude of the bead lines relative to the fluid $B / D$ for various values of bead diameter range $D$ and of frequency $f$.

rather from the competition between an attraction between two adjacent lines at large distance and a repulsion between them at short distance.

We first made sure that eventual magnetic interactions between the bronze beads and the electro-magnetic shaker were not involved in this ordering phenomenon by reproducing the same pattern using glass beads. Moreover, the high electrical conductivity of water prevents electrostatic repulsive interactions between beads.

In the case of an analogous periodic order occurring in suspensions under shear flow at low Re as reported in Ref. 4, the alignment of beads, as well as a repulsive hydrodynamic interaction between them, are attributed to the steady flow induced by the oscillating rotation of beads under the effect of the oscillating shear stress. ${ }^{4,17}$ Fluid driven into rotation by the bead is expelled away from the bead in its equatorial plane because of centrifugal force. Consequently, fluid is sucked towards the bead along its axis of rotation due to volume conservation. The polar suction induces attraction between the beads perpendicular to the direction of oscillation, leading to formation of lines of beads. The equatorial expulsion induces repulsion along the direction of oscillation, explaining the repulsion between the lines of beads. This flow is drawn in Fig. 9(a). In their experiment, the au- thors of Ref. 4 observed that $\lambda$ scales as $D$ but does vary neither with $\operatorname{Re}$ around $\operatorname{Re}=10^{-3}$, nor with $\phi_{S}$ in a large range $\phi_{S}=0.1-0.55$. No mechanism is proposed for a possible attraction between lines that could explain the independence of $\lambda$ with respect to $\phi_{S}$. We mention these explanations given for low Reynolds hydrodynamics since they rely on nonlinear effects of nonzero time-averaged value which can be of large magnitude in our experiment. In particular, the above proposed mechanism of line formation could be the same as in our experiment since the beads rotate alternatively due to solid and fluid friction.

In their review on streaming flows, ${ }^{18}$ Petit and Gondret also mention a mechanism proposed by König and cited by Rayleigh ${ }^{19}$ explaining the formation of similar periodic pattern observed at higher Re in powder at the antinodes of the standing acoustic wave in the Kundt's tube experiment, as presented in the Introduction. ${ }^{9}$ The particles are supposed to be sufficiently dense to behave as obstacles for the flow. The alternating inertial flow is shrinked and accelerated between two neighboring particles aligned normal to the flow, thus the mean pressure between the particles is lower than the mean pressure far from them: They attract and constitute lines perpendicular to the oscillating flow [see Fig. 9(b)]. On the contrary, the inertial flow is slower in the wake between two neighboring particles aligned along the flow direction, thus the mean pressure is higher between the particles than far from them: They repel [see Fig. 9(c)]. Although this mechanism is valid for translational motion of the particles relative to the fluid and for high Re, no mechanism is proposed of an attraction between lines at large distance. Finally, we mention apparently opposite theoretical predictions given by Tabakova and Zapruanov, ${ }^{20,21}$ i.e., a repulsion between beads in the plane normal to the flow and an attraction along the flow direction.

A strinking feature of the streaming flow around a cylinder or a sphere oscillating in an unbounded fluid is the quadrupolar vorticity distribution around the object. Theories valid for $B / D \ll 1$ predict that when the Strouhal number $D^{2} \omega / v$ is much smaller than unity, these four steady vorticies [represented in Fig. 10(a)] suck the fluid towards the moving object in the flow direction and eject the fluid away from it in the plane normal to the flow. ${ }^{10,18}$ For $D^{2} \omega / v \gg 1$, these vortices are reversed, i.e., suck the fluid towards the moving object in the plane normal to the flow and eject the fluid away from it in the flow direction (four smaller vortices of opposite direction of flow, located in the viscous boundary layer near the obstacle, also remain, as drawn in Fig.

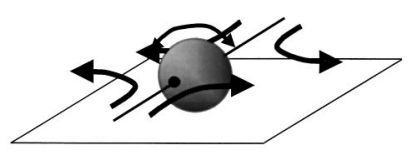

(a)

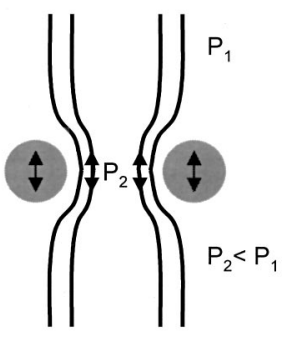

(b)

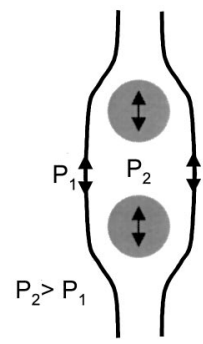

(c)
FIG. 9. (a) Ejection of fluid in the equatorial plane of a rotating sphere correlated to suction of the fluid along its axis of rotation. (b) Average pressure drop (under assumption of inertial flow) between two oscillating spheres aligned in the direction of vibration. (c) Average overpressure (under assumption of inertial flow) in the wake between two oscillating spheres aligned with the direction of vibration. 


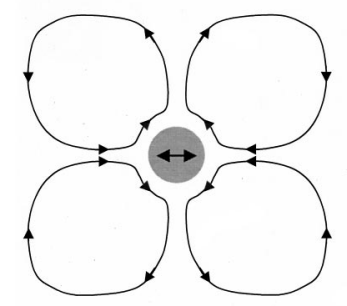

(a)

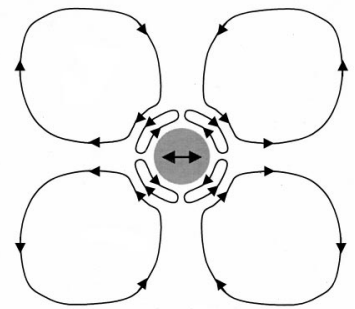

(b)

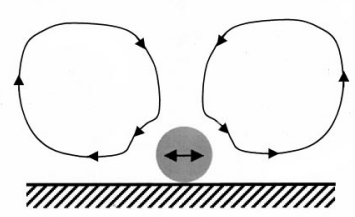

(c)
FIG. 10. Quadrupolar vorticity distribution around an oscillating cylindersphere (a) at low and (b) high Strouhal number. (c) Hypothetical vorticity distribution around a sphere oscillating close to a wall at high Re.

10(b). ${ }^{22-24}$ This inversion of the direction of rotation of the quadrupolar distribution of steady vortices (called hereafter "flow transition") should occur $D^{2} \omega / v$ being of the order of unity. From an experimental point of view, the flow transition is found by Petit and Gondret ${ }^{18}$ to occur in the range $5<D^{2} \omega / v<14$, whereas Andres and Ingard find that the Reynolds number $\operatorname{Re}=\left(D^{2} \omega / v\right)(B / D)$ better defines the exact limit between the two regimes, the parameter $B / D$ having a small but actual influence on this limit, even when $B / D$ $>1 .{ }^{10}$ Considering first $D^{2} \omega / v$ as the parameter driving the flow transition, we note that in our experiment the periodic pattern was observed for $D^{2} \omega / v>5.9$, a value compatible with the measurements of the flow transition done by Petit and Gondret. ${ }^{18}$ Using this criterion, the periodic pattern seems to appear when vortices eject fluid along the direction of the flow. Considering now Re as the parameter driving the flow transition, as proposed by Andres and Ingard, we compare in Fig. 11 the values of $\operatorname{Re}$ and $B / D$ at which the periodic pattern was observed in our experiment to the experimental values reported in Ref. 10 at which the flow transition occurs around a cylinder and a sphere in a practically unbounded fluid. Obviously, the occurrence of the periodic pattern cannot be linked to the flow transition around the cylinder. The comparison between the flow transition line for the isolated sphere in an unbounded fluid and our experiment is impossible because the flow transition line for the isolated sphere was not determined at high enough values of $B / D$.

As a conclusion of this part, there may exist a correlation between the occurrence of the periodic pattern and the existence of steady vortices ejecting fluid along the flow direction, inducing line repulsion. Anyway, the above comparisons have to be considered with caution, since there may be large discrepancies between the flow around an isolated sphere in an unbounded fluid and the flow around an array of spheres touching a wall. Moreover, the role of the rotation of the sphere and of neighboring spheres was not considered. An experimental determination of the steady flow around a sphere near a wall is strongly needed in order to confirm or reject the hypothesis concerning the flow represented in Fig.

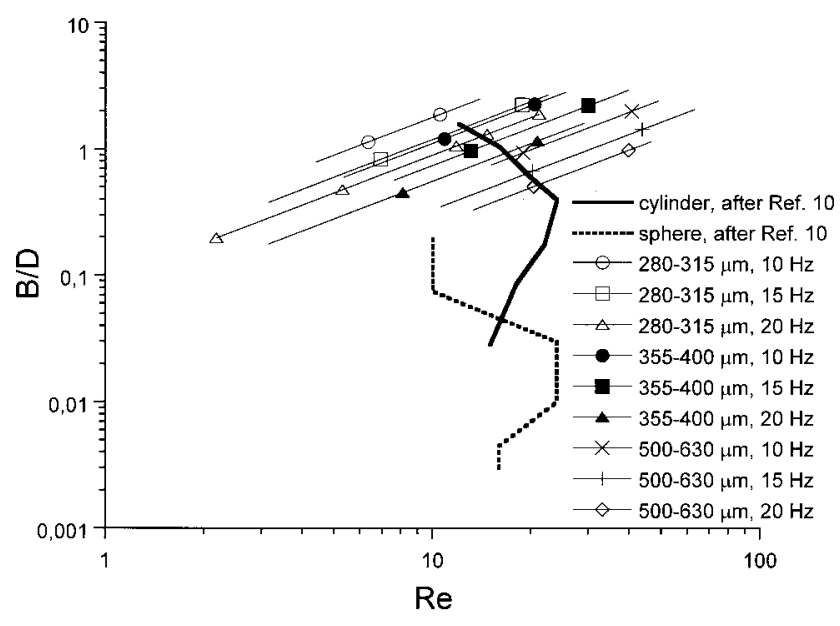

FIG. 11. Experimental diagram describing the regimes of flow around a cylinder-sphere oscillating in an unbounded fluid, after Ref. 10: The continuous (resp. dashed) line separates the left-hand region of the diagram where vortices suck the fluid towards the cylinder (resp. sphere) along the direction of oscillations [see Fig. 10(a)] from the right-hand region where vortices expel the fluid away from the cylinder (resp. sphere) along the direction of oscillations [see Fig. 10(b)]. The lines decorated with symbols are the parameter sets for which the periodic pattern was observed in our experiment.

10(c). Nevertheless, we have no mechanism explaining the attraction between the bead lines at large distance. It is also noteworthy that up to now no theoretical approach could deal with $B / D>1$, nor with $\mathrm{Re} \simeq 1$, which is precisely the case in our experiments. We finally mention that the steady streaming over a wavy wall as studied by Kaneko and Honji ${ }^{25}$ may resemble the flow between the lines of beads. Since their experiment was dedicated to the study of the mechanism of formation of submarine sand dunes, the question whether the occurrence of the steady pattern is linked to a flow transition at a finite value of any control parameter concerns not only our experiment but also the initial mechanism of formation of sand dunes (called "rolling grain" ripples).

\section{QUALITATIVE REMARKS ON PATTERN FORMATION AND DEFECTS}

As the shaker is switched on, the transient leading to the steady pattern and its duration depends strongly on the vibrational parameters $A$ and $f$, as well as on the initial bead distribution. When this initial distribution is a square [see Fig. 12(a)], lines of beads form first along its both sides parallel to the direction of vibrations, then lengthen slowly [see Fig. 12(b)]. In this case of two-dimensional initial beads distribution, the mechanism of pattern formation is a lengthening of the lines through their global translation away from the square perpendicular to the direction of vibrations. In particular, the distance between the lines remains constant during their lengthening. When the initial bead distribution is a stripe [see Fig. 12(c)], thick bead lines (i.e., more than one bead in thickness) separate from the stripe and drift away, giving space to other lines to separate from the stripe or to divide themselves in thinner lines [see Fig. 12(d)]. In that case of one-dimensional initial beads distribution, the mechanism of pattern formation is rather a decompaction of 


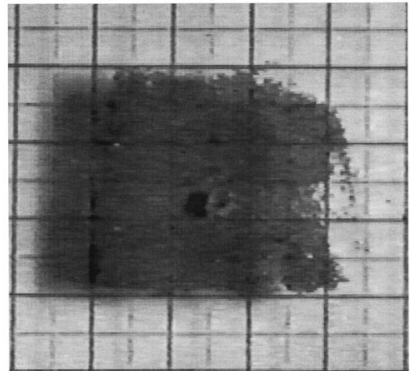

(a)

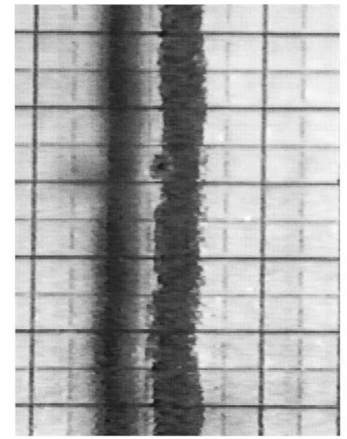

(c)

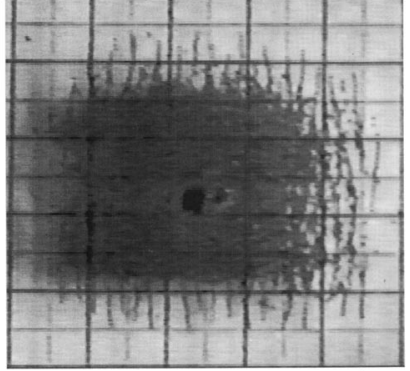

(b)

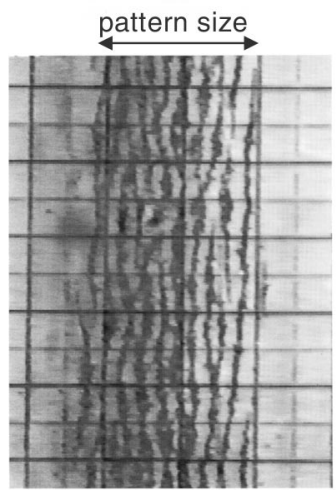

(d)
FIG. 12. (a) Initial dense square distribution of $D=355-400 \mu \mathrm{m}$ beads. (b) Distribution of beads $5.0 \mathrm{~s}$ after $f=20 \mathrm{~Hz}, \Gamma=14 \mathrm{~m} \cdot \mathrm{s}^{-2}$ vibrations have been switched on. (c) Initial dense stripe distribution of $D=355-400 \mu \mathrm{m}$ beads. The stripe visible on the left hand side of the picture is the shadow on the diffusing surface of the bead stripe visible on the right hand side of the picture. (d) Distribution of beads $4.3 \mathrm{~s}$ after $f=20 \mathrm{~Hz} \Gamma=18.7 \mathrm{~m} \cdot \mathrm{s}^{-2}$ vibrations have been switched on.

the stripe through the dilation of the pattern (i.e., a continuous increase of the distance between the bead lines). The evolution in time of the distance between the most distant lines of the pattern formed from a stripe [the "size" of the pattern, as defined as in Fig. 12(d)] is plotted in Fig. 13 for $D=355-400 \mu \mathrm{m}, f=20 \mathrm{~Hz}$, and $\Gamma=18.7 \mathrm{~m} \cdot \mathrm{s}^{-2}$. This evolution is slow and cannot be simply characterized by a relaxation time. A time scale which would be more characteristic of the interaction between two neighboring bead lines is the characteristic time of formation of the first (thick) line from the initial stripe. The evolution in time of the distance between the first line and the stripe is plotted in the inset of Fig. 13 for $D=355-400 \mu \mathrm{m}, f=20 \mathrm{~Hz}$, and $\Gamma=18.7 \mathrm{~m} \cdot \mathrm{s}^{-2}$. The associated time scale is very short, of the order of a tens of vibration periods. A more rapid image acquisition is needed to measure it precisely as a function of $A, D$, and $f$.

We finally mention that the steady pattern often exhibits cristalline defects which consist in fork defects, clusters of beads which result in line thickening, and local pattern compression-dilation, all these defects being pointed out in Fig. 2. These defects, which arise from bead diameter or shape inhomogeneities and from interaction of the lines with boundaries, evolve slowly in time. They are responsible for the dispersion of the $\lambda$ measurements.

\section{CONCLUSION}

We first summarize our results. Under the effect of horizontal vibrations, an 2D assembly of beads heavier than the

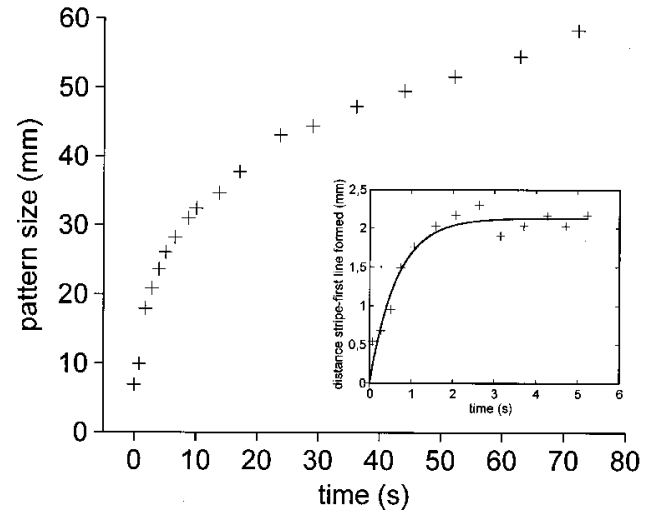

FIG. 13. Evolution in time of the pattern size [as defined in Fig. 12(b)] of $D=355-400 \mu \mathrm{m}$ beads from an initial dense stripe distribution under the effect of $f=20 \mathrm{~Hz}, \Gamma=14 \mathrm{~m} \cdot \mathrm{s}^{-2}$ vibrations applied from $t=0$. After $t$ $=80 \mathrm{~s}$, the pattern becomes 2D. Inset: Evolution in time of the distance between the line formed first and the stripe. The line is the best fit of the form $\lambda[1-\exp (-t / \tau)]$ with $\tau=0.64 \mathrm{~s}$.

surrounding fluid is ordered in one bead thick lines, regularly spaced, perpendicular to the direction of vibrations. The amplitude $B$ of the oscillations of the beads relative to the fluid varies linearly with the amplitude $A$ of the oscillations of the fluid. A simple phenomenological model based on dynamic solid friction and viscous drag gives qualitatively account for this linear dependence; as a consequence, the beads probably slip most of the time on the vessel wall. The period $\lambda$ of the pattern is found to vary with the bead diameter $D$, oscillation frequency $f=\omega / 2 \pi$, and viscosity $v$, according to the empirical law $\lambda / D \sim(B / D)^{0.5}(B \omega D / v)^{-0.21}$. The onset of formation of the periodic pattern may be linked to the formation of steady vortices (streaming flow) explaining the formation of lines of beads and repulsion between lines.

We now come back to the comparison between the pattern observed in the Kundt's tube experiment and our observations. In the Kundt's tube experiment, the periodic pattern is visible only where the displacement of air is large. Petit and Gondret ${ }^{18}$ report that the distance between two neighboring lines decreases with increasing distance of these lines to the antinode, i.e., with decreasing air oscillation amplitude. This is in agreement with our measurements which show that $\lambda$ increases with increasing $B$, which itself increases with increasing $A$. The main difference between our experimental configuration and the Kundt's tube experiment is that the bottom wall moves with the fluid, whereas the wall of the Kundt's tube is motionless. Providing that the bead rotation has no pertinent effect on the interaction between the lines, this difference should actually influence only the relation between $B$ and $A$. Despite the fact that the streaming flows around bluff bodies were mainly studied using compression waves ${ }^{26}$ the wavelength of the acoustic waves are always large compared to the body. Thus, in acoustic streaming flow studies, the fluid can be considered as oscillating with an homogeneous amplitude around the body, as in our experiment. Very recently, Gollub observed a dynamic crystallization of beads on a hexagonal 2D lattice when the same suspension as our was vibrated vertically. Since the measured lattice constant of the pattern was found to be larger than the 
bead diameter, this pattern also results from a competition between attractive forces between oscillating beads at large distance and repulsive forces at small distance.

Perspectives of this work are numerous. Further experiments are needed in order to determine the alternating and streaming flows around an oscillating-rotating sphere near a wall. A test of validity of the empirical scaling law for $\lambda$ at higher frequencies and other values of viscosity and density constrast should also be done. Finally, since this ordering phenomenon could be applied to micro-deposition as well as composite materials, its microscopic limit is of technological interest and should be investigated.

\section{ACKNOWLEDGMENTS}

R.W. thanks CNES for financial support. The authors thank C. Bacon (Laboratoire de Mécanique Physique, Bordeaux-I University), Y. Joyeux (Centre de Recherche Paul Pascal), F. Palencia, and C. Lecoutre (Institut de Chimie de la Matière Condensée) for technical support and assistance during the preliminary experiments. F. Feuillebois (Laboratoire de Physique Thermique, Ecole Supérieure de Physique et Chimie Industrielle, Paris) and S. Fauve (Laboratoire de Physique Statistique) are also thanked for fruitful discussions and for having pointed out relevant references.

${ }^{1}$ L. Petit, "Ecoulement et mise en structure de suspensions macroscopiques," Ann. Phys. (Paris) 16, 155 (1991).

${ }^{2}$ R. L. Hoffman, "Discontinuous and dilatant viscosity behavior in concentrated suspensions. I. Observation of a flow instability," Trans. Soc. Rheol. 16, 155 (1972).

${ }^{3}$ N. A. Clark, A. J. Hurd, and B. J. Ackerson, "Single colloidal crystals," Nature (London) 281, 57 (1979).

${ }^{4}$ L. Petit and B. Noetinger, "Shear-induced structures in macroscopic dispersions," Rheol. Acta 27, 437 (1988).

${ }^{5}$ D. Leighton and A. Acrivos, "The shear induced migration of particles in concentrated suspensions," J. Fluid Mech. 181, 415 (1987).

${ }^{6}$ C. J. Chow, S. W. Sinton, J. H. Iwamiya, and T. S. Stephens, "Shearinduced migration in Couette and parallel-plate viscometers: NMR imaging and stress measurements," Phys. Fluids 6, 2561 (1994).
${ }^{7}$ M. Tirumkudulu, A. Tripathi, and A. Acrivos, "Particle segregation in monodisperse sheared suspensions," Phys. Fluids 11, 507 (1999); 11, S13 (1999).

${ }^{8}$ M. Tirumkudulu, A. Mileo, and A. Acrivos, "Particle segregation in monodisperse sheared suspensions in a partially filled rotating horizontal cylinder," Phys. Fluids 12, 1615 (2000).

${ }^{9}$ E. N. Andrade and S. K. Lewer, "New phenomena in a sounding dust tube," Nature (London) 124, 724 (1929).

${ }^{10}$ J. M. Andres and U. Ingard, "Acoustic streaming at low Reynolds numbers," J. Acoust. Soc. Am. 25, 932 (1953).

${ }^{11}$ M. R. Maxey and J. J. Riley, "Equation of motion for a small rigid sphere in a nonuniform flow," Phys. Fluids 26, 883 (1983).

${ }^{12}$ A. J. Goldman, R. G. Cox, and H. Brenner, "Slow viscous motion of a sphere parallel to a plane wall.-I Motion through a quiescent fluid," Chem. Eng. J. 22, 637 (1967).

${ }^{13}$ G. G. Stokes, "On the effect of internal friction of fluids on the motion of pendulum," Trans. Cambridge Philos. Soc. 9, 8 (1851).

${ }^{14} \mathrm{~F}$. Feuillebois and A. Lasek, "On the rotational historic term in nonstationary Stokes flow,” Q. J. Mech. Appl. Math. 23, 435 (1977).

${ }^{15}$ J. R. Smart, S. Beimfohr, and D. Leighton, Jr., "Measurement of the translational and rotational velocities of a noncolloidal sphere rolling down a smooth inclined plane at low Reynolds number,' Phys. Fluids A 5, 13 (1993).

${ }^{16}$ F. P. Bowden and D. Tabor, The Friction and Lubrification of Solids (Clarendon, Oxford, 1950 and 1964), Vols. I and II.

${ }^{17}$ P. Gondret, Ph.D. thesis, Lyon-I University (1994).

${ }^{18}$ L. Petit and P. Gondret, "Redressement d'un écoulement alternatif," J. Phys. II France 2, 2115 (1992).

${ }^{19}$ J. W. S. Lord Rayleigh, The Theory of Sound (Dover, New York, 1945).

${ }^{20}$ S. S. Tabakova and Z. D. Zapruanov, "On the hydrodynamic interaction of two spheres oscillating in a viscous fluid.-I. Axisymetrical case," ZAMP 33, 344 (1982).

${ }^{21}$ S. S. Tabakova and Z. D. Zapruanov, "On the hydrodynamic interaction of two spheres oscillating in a viscous fluid.-II. Three dimensional case," ZAMP 33, 487 (1982).

${ }^{22}$ E. N. Andrade, "On the circulation caused by the vibration of air in a tube," Proc. R. Soc. London, Ser. A 134, 447 (1931).

${ }^{23}$ H. Schlichting, "Berechnung ebener periodischer Grenzschichtrömungen," Phys. Z. 33, 327 (1932).

${ }^{24}$ H. Schlichting, Boundary-layer Theory, 7th ed. (McGraw-Hill, New York, 1979).

${ }^{25}$ A. Kaneko and H. Honji, "Double structures of steady streaming in the oscillatory viscous flow over a wavy wall," J. Fluid Mech. 93, 727 (1979).

${ }^{26}$ W. M. Nyborg, in Physical Acoustics, Principles and Methods, edited by W. P. Mason (Academic, New York, 1965), Vol. II, Part b, Chap. 11, pp. 265-331. 